\title{
Fallout from the ORBITA trial - is angioplasty in a spin?
}

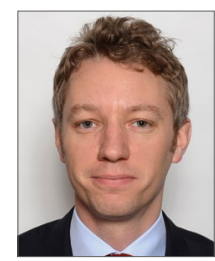

Robert A. Byrne*, MB, BCh, PhD, Deputy Editor

In the immediate aftermath of the Transcatheter Therapeutics meeting in Denver, CO, USA, one trial seemed to dominate the discussions, at least on social media platforms. Within one day of publication, the trial report of the Objective Randomised Blinded Investigation with optimal medical Therapy of Angioplasty in stable angina (ORBITA) trial published in The Lancet ${ }^{1}$ had already been mentioned on Twitter 849 times. To put this into perspective, the SENIOR trial presented and published around the same time in The Lancet ${ }^{2}$ was mentioned 21 times. The report received widespread attention in the lay media, with one commentator concluding that "a procedure used to relieve chest pain in hundreds of thousands of heart patients each year is useless for many of them"3. Opinions in the medical literature have been polarised, a sign, sometimes, of a study that is asking the right question. However, some physicians went so far as to suggest that the results of this trial represent the final nail in the coffin of percutaneous coronary intervention (PCI $)^{4}$. As the dust from ORBITA settles, are we still in a spin or is there light on the horizon?

The ORBITA trial was a modest-sized, multicentre, investigator-initiated, independently funded, randomised trial enrolling selected patients with symptoms of angina, preserved left ventricular function and single-vessel coronary disease. All patients underwent a run-in period of six weeks, during which medical therapy was optimised. At the end of this time, patients were taking a mean number of antianginal medications of around 3.0 and had a Seattle Angina Questionnaire physical limitation score of around 70, indicating low-to-moderate limitation. Following this, 200 patients were randomly allocated to PCI or to continued medical therapy. All patients underwent invasive physiological measurements of the study lesion, and both patients and referring physicians were blinded to whether or not the patient received PCI. The patients wore headsets and received sedation to maintain blinding. For these reasons, the control group was referred to as having received placebo therapy. The primary endpoint was change in treadmill exercise time between randomisation and follow-up at six weeks. The main finding was that there was no significant difference in change in exercise time between the groups and that the null hypothesis could not be rejected. Treadmill exercise time increased by $28.4 \mathrm{~s}$ after PCI (from $528.0 \pm 178.7$ to $556.3 \pm 178.7 \mathrm{~s}$ ) in the PCI group and by $11.8 \mathrm{~s}$ (from $490.0 \pm 195.0$ to $501.8 \pm 190.9 \mathrm{~s})$ in the placebo group $(\mathrm{p}=0.20)$.

The authors should be congratulated for conducting an interesting clinical trial and for presenting the results in a considered manner. In keeping with recommendations for increased research transparency ${ }^{5}$, the trial protocol was submitted for peer review and a summary published in advance on a publicly accessible website $^{6}$. Angiographic images from each patient studied were made available in the supplementary material. The internal validity of the study appears to be high in many respects. Methods used for randomisation sequence generation and allocation concealment were appropriate, blinding appeared to have been successful and independent data monitoring was carried out. The primary endpoint was appropriate for the study hypothesis under test. Analysis of secondary endpoints largely supported the findings of the primary endpoint analysis, with the exception of peak stress wall motion on dobutamine stress echo, which favoured the PCI group. 
At the same time, the trial results must be interpreted against the background of some important limitations. From a technical point of view, two issues related to the primary endpoint analysis should be considered. First, the power of the study to detect a difference between the groups was lower than planned. When comparing mean values of continuous variables like exercise time, the standard deviation of the observations is an important consideration in calculating the sample size required. In ORBITA, the observed between-patient standard deviation was more than twice as high as that assumed, resulting in a power to detect a difference between the groups - under otherwise unchanged assumptions of perhaps as low as $20 \%$. Second, comparing changes in a parameter such as exercise time is fraught with difficulty when the starting position is different in both groups. In ORBITA, despite random treatment allocation, mean exercise time was 38 seconds higher at baseline than in the PCI group. Moreover, the lack of difference between the groups in terms of exercise time was due in large part to a subsequent increase in exercise time in the control group. The authors interpret this as evidence of a placebo effect. In actual fact, the lower exercise time in the control group at baseline is likely due to chance, and the subsequent improvement with repeat testing is an expected phenomenon. Statistically speaking this is known as regression to the mean and failure to recognise this phenomenon is not an infrequent reason to misinterpret clinical trial results?

In addition, a number of factors limit the external validity of the observations. First, the follow-up period was very short - six weeks. The clinical success of this strategy and acceptance from a patient preference standpoint, over a longer time horizon is unclear. Indeed, a much larger, multicentre trial comparing PCI with medical therapy with follow-up out to three years was also presented at the same meeting in Denver and showed a more than $50 \%$ reduction in major adverse cardiac events with PCI over this time period ${ }^{8}$. Second, whether the intensive, consultant physician-led patient-care model used to optimise medical treatment in ORBITA is feasible outside of the setting of a carefully controlled clinical trial is not known. Third, the findings apply to selected patients with only a small minority of patients planned for PCI at five centres over almost four years enrolled in the trial. In addition, patients with multivessel disease or reduced left ventricular function are not represented. Indeed, there was only a single adverse cardiac events in the entire study cohort during follow-up. These factors limit the clinical relevance of the findings for the patients we treat day in, day out.

The ORBITA trial sheds important light on the potential for benefit in patients with single-vessel coronary disease, managed with aggressive medical therapy and close supervision over a limited time frame. Details on longer-term follow-up of the included patients will be awaited with interest. The observations of the investigators might provide a rationale for a larger clinical trial focused on clinical outcomes, though whether such a trial will be performed remains an open question. Failing that, the data generated will continue to inform our discussions on the topic for the foreseeable future. Headline writers and Twitter handles require a limited number of characters to summarise the findings of a clinical trial. The reality of the data presented by the ORBITA investigators is much more nuanced but no less interesting.

\section{References}

1. Al-Lamee R, Thompson D, Dehbi HM, Sen S, Tang K, Davies J, Keeble T, Mielewczik M, Kaprielian R, Malik IS, Nijjer SS, Petraco R, Cook C, Ahmad Y, Howard J, Baker C, Sharp A, Gerber R, Talwar S, Assomull R, Mayet J, Wensel R, Collier D, Shun-Shin M, Thom SA, Davies JE, Francis DP; ORBITA investigators. Percutaneous coronary intervention in stable angina (ORBITA): a double-blind, randomised controlled trial. Lancet. 2017 Nov 1. [Epub ahead of print].

2. Varenne O, Cook S, Sideris G, Kedev S, Cuisset T, Carrié D, Hovasse T, Garot P, El Mahmoud R, Spaulding C, Helft G, Diaz Fernandez JF, Brugaletta S, Pinar-Bermudez E, Mauri Ferre J, Commeau P, Teiger E, Bogaerts K, Sabate M, Morice MC, Sinnaeve PR; SENIOR investigators. Drug-eluting stents in elderly patients with coronary artery disease (SENIOR): a randomised single-blind trial. Lancet. 2017 Oct 31. [Epub ahead of print].

3. Kolata G. 'Unbelievable': Heart Stents Fail to Ease Chest Pain. The New York Times. 2017 Nov 2. https://www.nytimes. com/2017/11/02/health/heart-disease-stents.html

4. Brown DL, Redberg RF. Last nail in the coffin for PCI in stable angina? Lancet. 2017 Nov 1. [Epub ahead of print].

5. Steps towards transparency in research publishing. Nature. 2017;549:431.

6. Protocol 15PRT/06897: Objective Randomised Blinded Investigation with optimal medical Therapy of Angioplasty in stable angina (ORBITA trial) - NCT02062593. Available at: http://www.thelancet.com/protocol-reviews-list (last accessed 10.11.2017).

7. Morton V, Torgerson DJ. Effect of regression to the mean on decision making in health care. BMJ. 2003;326:1083-4.

8. Fearon WF, Nishi T, De Bruyne B, Boothroyd DB, Barbato E, Tonino P, Jüni P, Pijls NHJ, Hlatky MA; FAME 2 Trial Investigators. Clinical Outcomes and Cost-Effectiveness of Fractional Flow Reserve-Guided Percutaneous Coronary Intervention in Patients With Stable Coronary Artery Disease: Three-Year Follow-Up of the FAME 2 Trial (Fractional Flow Reserve Versus Angiography for Multivessel Evaluation). Circulation. 2017 Nov 2. [Epub ahead of print]. 